\title{
ENTERPRISE RISK MANAGEMENT E 0 DESEMPENHO EMPRESARIAL: UMA PERSPECTIVA CONTINGENCIAL
}

\section{ENTERPRISE RISK MANAGEMENT AND ORGANIZATION PERFORMANCE: A CONTINGENTIAL PERSPECTIVE}

\author{
"O artigo foi aprovado e apresentado no XVIII USP International Conference in Accounting, realizado de 25/07 a 27/07 de 2017, em São Paulo (SP) "
}

\begin{abstract}
RESUMO
Este estudo teve por objetivo verificar a relação entre Enterprise Risk Management (ERM) e as variáveis contingenciais mediante o desempenho empresarial da organização. Para tal, foi realizada uma pesquisa descritiva e documental, com abordagem quantitativa em empresas listadas na B3. Os dados coletados compreendem o período de 2012 a 2016. A amostra do estudo constituiu-se de todas as empresas brasileiras que apresentaram todos os dados necessários para a análise no período avaliado, o que correspondeu a 311 empresas. As métricas utilizadas para a mensuração do ERM, variáveis contingenciais e o desempenho empresarial foram obtidas no banco de dados Economática ${ }^{\circledR}$ e formulários de referências no site da B3. Posteriormente, com base no cálculo das variáveis analisadas, utilizou-se a técnica estatística Cluster Analysis para identificar as empresas com alto desempenho. Na sequência, realizou-se regressão de dados em painel. Os resultados sugerem que empresas de alto desempenho atribuem maior importância às variáveis contingenciais do que as demais organizações, quando da implementação do ERM. Os resultados do estudo demonstram algumas fragilidades, tendo em vista que as variáveis contingenciais complexidade empresarial, tamanho da empresa e monitoramento do conselho de administração não foram significantes no modelo. Contudo, pode-se concluir por meio dos resultados obtidos que empresas de alto desempenho atribuem maior importância às variáveis contingenciais do que as demais organizações, quando da implementação do ERM.
\end{abstract}

Palavras chave: Gestão de Risco. Fatores Contingenciais. Enterprise Risk Management. Desempenho Empresarial.

\begin{abstract}
The objective of the study is to verify the relationship between Enterprise Risk Management (ERM) and contingency variables through the organization performance. A descriptive and documentary research with quantitative approach was performed in companies listed in B3. The data collected cover the period from 2012 to 2016. The study sample consisted of all Brazilian companies that presented all the necessary data for the analysis in the evaluated period, which corresponded to 311 companies. For the ERM measurement, contingency variables and business performance were obtained from the Economática database and reference forms from the B3 website. Then, based on the calculation of the variables analyzed, the Cluster Analysis statistical technique was used to identify the companies with higher performance. For the ERM measurement, contingency variables and business performance were obtained from the Economática ${ }^{\circledR}$ database and reference forms from the B3 website. Then, based on the calculation of the variables analyzed, the Cluster Analysis statistical technique was used to identify the companies with high performance. Subsequently, panel data regression was performed. The results suggest that high performance companies attach more importance to contingency variables than other organizations when implementing ERM. The study results demonstrate some weaknesses, considering that the contingency variables business complexity, company size and board monitoring were not significant in the model. However, it can be concluded from the results obtained that high performance companies attach greater importance to contingency variables than other organizations when implementing ERM.
\end{abstract}

Keywords: Risk Management. Contingency Factors. Enterprise Risk Management. Organization performance.
Fellipe Jacomossi

Doutorando do Programa de PósGraduação em Contabilidade da Universidade Regional de Blumenau - FURB; Mestre em Ciências Contábeis pela Universidade Regional de Blumenau - FURB; Graduação em Administração (2015), Ciências Contábeis (2013) e Direito (2011) pelo Centro Universitário de Brusque - UNIFEBE. Endereço: Rua Oswaldo Heil, 08, Nova Brasília, Brusque, SC, CEP: 88.352-660. E-mail: gerencia@ dinamicacontabilidade.com.br. Telefone: 55(47) 3321-0565

\section{Micheli Aparecida Lunardi Doutoranda do Programa de Pós- Graduação em Contabilidade da Universidade Regional de Blumenau - FURB; Mestre em Ciências Contábeis pela Universidade Regional de Blumenau - FURB; Especialista em Controladoria e Finanças pela Universidade do Estado de Santa Catarina - UDESC; Graduação em Ciências Contábeis pela Universidade do Estado de Santa Catarina - UDESC. Endereço: Rua Antônio da Veiga, 140, Sala D-202, Campus I, Bairro Victor Konder, CEP 89030-903 - Blumenau, SC - Brasil. E-mail: micheli.lunardi@yahoo. com.br Telefone: 55(47) 3321-0565}
Marcia Zanievicz da Silva
Professora do Programa de Pós- Graduação em Ciências Contábeis da Universidade Regional de Blumenau - FURB; Doutora em Contabilidade e Administração pela FURB; Mestre em Ciências Contábeis pela Universidade Federal de Santa Catarina - UFSC. Endereço: Rua Antônio da Veiga, 140, Sala D-202, Campus I, Bairro Victor Konder, CEP 89030-903 - Blumenau, SC - Brasil. E-mail: mzsilva@furb.br Telefone: 55(47) 3321-0565 


\section{INTRODUÇÃO}

A competitividade no século XXI desponta mudanças significativas no ambiente de negócios das organizações (SOIN; COLLIER, 2013; PELEIAS et al., 2013). Desta maneira, os gestores têm promovido alterações e desenvolvido mecanismos nas estruturas das organizações e nos processos operacionais, como forma de encarar os riscos e as incertezas do ambiente de negócios (LIEBENBERG; HOYT, 2003; TRAPP; CORRAR, 2005; BEASLEY; CLUNE; HERMANSON, 2005; PELEIAS et al., 2013), dentre estes mecanismos destacam-se aqueles voltados para avaliação e gerenciamento de riscos corporativos, os chamados Enterprise Risk Management (ERM).

O ERM é um processo sistemático, geralmente introduzido nas organizações por meio de um conselho de administração, sendo difundido entre todos os âmbitos empresariais. Aplica-se uma configuração estratégica e em toda a entidade, com intuito de identificar eventos que possam afetar a organização. Este gerenciamento busca enquadrar a empresa dentro do apetite de risco de seus gestores, fornecendo garantias razoáveis quanto à realização dos objetivos da entidade (COHEN; KRISHNAMOORTHY; WRIGHT, 2017). Para Lechner e Gatzert (2017), o gerenciamento de riscos corporativos tornou-se relevante nos últimos anos, devido a uma crescente complexidade dos riscos e ao desenvolvimento de estruturas regulatórias.

Tendo identificado os principais riscos da empresa, o ERM deve encontrar uma maneira consistente de medir a exposição da empresa a esses riscos através de uma abordagem comum, que possa ser usada para identificar e quantificar as exposições significativas da organização (NOCCO; STULZ, 2006). O gerenciamento de risco corporativo exige a criação de controles e sistemas com o intuito de tornar a empresa mais resiliente e adaptável às principais mudanças ocorridas no ambiente externo (DICKINSON, 2001). Ainda, a abordagem do ERM requer que os gestores identifiquem e gerenciem os riscos em uma perspectiva corporativa holística, em detrimento de uma perspectiva restritiva ou departamentalizada (LIEBENBERG; HOYT, 2003; KLEFFNER; LEE; MCGANNON, 2003; BROMILEY et al., 2015).

As organizações estão implementando ERM para aumentar a efetividade de suas atividades, com o objetivo final de aumentar o valor para os seus stakeholder (BEASLEY; CLUNE; HERMANSON, 2005). Segundo Nocco e Stulz (2006), em uma empresa onde o risco é bem compreendido, a possibilidade de obtenção de recursos por meio de seu possível gerenciamento é maximizada, tendo em vista a confiança gerada aos investidores. Embora existam evidências que demonstram que o gerenciamento dos riscos corporativos pode aumentar ou diminuir o valor para o acionista, esses motivos dependem, também das características individuais de cada empresa (BEASLEY; PAGACH; WARR, 2008), bem como, as variáveis contingenciais em que estão expostas (GORDON; LOEB; TSENG, 2009).

O gerenciamento de riscos em algumas organizações consiste apenas em controlar o negócio para o cumprimento dos limites e políticas de risco, enquanto que em outras, a função é auxiliar a organização a conhecer as incertezas em seu ambiente competitivo (MIKES; KAPLAN, 2013). Embora os benefícios potenciais para o desempenho e o valor da empresa tenham levado as organizações a adotar sistemas de ERM (BEASLEY; PAGACH; WARR, 2008; HOYT; LIEBENBERG, 2011), ainda há pouca evidência sobre os benefícios do gerenciamento de riscos (BAXTER et al. 2013), bem como a interferência das variáveis contingenciais neste contexto.

Segundo Gordon, Loeb e Tseng (2009) a relação do desempenho empresarial e o ERM depende da correspondência adequada entre o sistema ERM e os fatores contingências. Para os autores, os cinco fatores que possuem um impacto na relação do desempenho empresarial e ERM são: incerteza ambiental, competição da indústria, complexidade da empresa, tamanho da empresa e monitoramento do conselho de administração (GORDON; LOEB; TSENG, 2009).

Frente as diversas contingências a serem monitoradas pelos sistemas de ERM, Gordon, Loeb e Tseng, (2009) desenvolveram um índice para medir a qualidade dos sistemas de gestão de risco das organizações, que fora denominado Enterprise Risk Management Index (ERMI). Os autores observaram a relação de tal índice com diversas variáveis contingenciais em empresas americanas. No entanto, não identificou-se no cenário brasileiro, a relação entres as variáveis contingenciais e o índice de gestão de risco.

Estudos demonstraram que a gestão de risco corporativo tende a causar um impacto significativo e positivo no valor e desempenho da empresa, todavia existem evidências divergentes (COHEN; KRISHNAMOORTHY; WRIGHT, 2017). Além disso, as pesquisas sobre gerenciamento de risco corporativo geralmente se concentram em indústrias específicas (HOYT; LIEBENBERG, 2011), não considerando a relação entre o gerenciamento de risco corporativo e o desempenho da organização mediante fatores contingenciais em que as empresas estão expostas. Desta maneira surge a seguinte questão de pesquisa: Qual a relação entre ERM e as variáveis contingenciais mediante o desempenho empresarial da organização? Assim, o objetivo deste estudo consiste em verificar a relação entre ERM e as variáveis contingenciais mediante o desempenho empresarial da organização.

As ondas de escândalos corporativos ampliam ainda mais a necessidade de as empresas sinalizarem seu compromisso com a gestão de riscos corporativos e o compliance, haja vista sua ampla gama de stakeholders (LIEBENBERG; HOYT, 2003). Estes escândalos fazem com que aumentem os padrões de prestação de contas frente aos conselhos e as partes interessadas, levando a um maior foco nos sistemas de ERM (KLEFFNER; LEE; MCGANNON, 2003). Mais amplamente, diz-se que o ERM promove uma maior conscientização dos riscos corporativos, o que facilita a tomada de decisão operacional e estratégica (HOYT; LIEBENBERG, 2011). Outra contribuição relevante da gestão de riscos para as organizações é agregar valor de forma sustentada às atividades da organização (PELEIAS et al. 2013), neste sentido, surgem cada vez mais estudos voltados ao exame da adoção do ERM e o seu impacto na gestão dos riscos. Todavia, tais pesquisas permanecem inconsis- 
tentes e inconclusivas, devido a uma especificação inadequada de como o gerenciamento de risco corporativo é usado na prática (MIKES; KAPLAN, 2013), gerando assim uma lacuna para realização de novas observações.

Embora muitos artigos envolvendo o ERM tenham surgido, a pesquisa acadêmica neste âmbito ainda está em fase embrionária (BROMILEY et al. 2015). Apesar de estudos empíricos terem observado a prevalência e a eficácia do ERM, poucos resultados significativos foram constatados, em grande parte devido às perspectivas de contingência em que as empresas estão expostas e que não são devidamente observadas (MIKES; KAPLAN, 2013).

Desta maneira, este paper é motivado a contribuir com a pesquisa empírica que busca examinar a relação entre o ERM e as variáveis contingenciais mediante o desempenho empresarial das organizações. O estudo também pretende contribuir com a literatura explorando como se altera o comportamento do desempenho mediante os fatores contingenciais aos quais as organizações estão expostas. Sendo assim, propõe-se que a gestão de riscos empresariais está relacionada com as variáveis contingenciais mediante o desempenho empresarial da organização.

\section{MODELO TEÓRICO DE ANÁLISE E HIPÓTESES DE PESQUISA}

O gerenciamento de risco empresarial, por meio de modelos que representam a estrutura organizacional, almeja desafiar as formas pré-existentes de conceber a incerteza, corrigindo possíveis falhas no ERM e os respectivos impactos resultantes sobre o desempenho (COSO, 2004). Através do ERM empresa avalia, controla, explora, financia e monitora riscos com o objetivo de aumentar o valor a curto e longo prazo da organização para suas partes interessadas (GORDON; LOEB; TSENG, 2009). Além disso, o ERM pode ser visto como uma importante ferramenta a ser utilizada pelos gestores no preparo das organizações frente às incertezas existentes no âmbito corporativo (ARENA; ARNABOLDI; AZZONE, 2010).

As organizações, visando enfrentar as incertezas ambientais, encorajam os gerentes a diferenciar os segmentos das empresas em relação ao ambiente de negócios (LAWRENCE; LORSCH, 1967; CHENHALL; MORRIS, 1986). Sendo assim, esses gerentes possuem autoridade para desenvolver seus próprios objetivos e estilos de gerenciamento, desenvolvendo competências para lidar com as incertezas ambientais (CHENHALL; MORRIS, 1986).

A literatura pressupõe que os altos níveis de incerteza percebida são prejudiciais ao desempenho organizacional, podendo ocorrer devido à crescente falta de previsão dos eventos futuros que afetam a entidade (BOURGEOIS, 1985). Todavia, organizações com bons sistemas de gestão de risco, ou seja, altos índices de ERMI (MIKES; KAPLAN, 2013) estão menos sujeitas a problemas frente às incertezas ambientais.

Para Gordon, Loeb e Tseng (2009) a incerteza ambiental cria dificuldades para as organizações devido à crescente falta de previsão dos eventos futuros. Assim, os riscos associados a uma empresa e a resposta apropriada a tais riscos provavelmente variam de acordo com a incerteza que enfrenta a empresa. No entanto, para os autores, empresas com alto desempenho (empresas bem sucedidas, avaliadas pelo alto índice dos indicadores ROE, ROA e MGL) está relação é mais forte entre a percepção ao risco e as incertezas ambientais (LIEBENBERG; HOYT, 2003). Desta maneira, elaborou-se a primeira hipótese da pesquisa:

\section{$\mathbf{H}_{1:}$ Há relação positiva entre ERMI e incerteza ambiental para as empresas de alto desempenho empresarial.}

Assim como a incerteza ambiental, a concorrência, principalmente entre organizações de um mesmo setor, gera a necessidade das organizações se apoiarem em sistemas de gerenciamento de riscos para tomada de decisão. Empresas de um mesmo nicho empresarial, acabam produzindo e fornecendo produtos e serviços similares, e assim, competindo por demanda de mercado (GE; MCVAY, 2005; DOYLE; GE; MCVAY, 2007).

Para Gordon, Loeb e Tseng (2009) existem muitas empresas de um setor que produzem e vendem produtos e serviços similares, gerando concorrência entre elas. A competição por vendas nas empresas com alta concorrência é muitas vezes acirrada, o que, por sua vez, significa que estas empresas enfrentam risco na realização de suas atividades.

No estudo de Khandwalla (1972), o autor examinou o efeito que o tipo de competição enfrentada pela organização tem sobre o uso dos controles de gestão. Concluiu que a sofisticação dos sistemas de contabilidade e controle foi influenciada pela intensidade da concorrência. Além disso, com base em uma análise de correlação, o autor constatou que a concorrência aumenta o uso de sistemas de controle de gestão de riscos sofisticados.

No que tange a concorrência entre as organizações e o gerenciamento de riscos corporativos, Meulbroek (2002) demonstrou que o aumento da concorrência influencia positivamente a ênfase do gerenciamento de riscos nas organizações, migrando suas ações de um foco defensivo para um mais ofensivo e estratégico. Além disso, a concorrência pode gerar pressão regulamentares e práticas gerenciais as quais tendem a impactar significativamente o ERM, quando a empresa possui alto desempenho (LIEBENBERG; HOYT, 2003; GORDON; LOEB; TSENG, 2009). Com base nestes pressupostos, a literatura apresenta indícios de que quanto maior o nível de concorrência frente as vendas de uma organização, mais competente deve ser o seu sistema de ERM, assim como, maior deve ser a adequação do ERM frente a concorrência setorial (GORDON; LOEB; TSENG, 2009). Desta forma, é delineada a segunda hipótese da pesquisa:

$\mathbf{H}_{2}$ : Há relação positiva entre ERMI e a concorrência setorial para as empresas de alto desempenho empresarial. 
A complexidade da empresa é outro fator geralmente observado por sistemas de gestão de risco. Segundo Gordon, Loeb e Tseng (2009), a complexidade da empresa é mensurada pela diversidade das transações comerciais realizadas. Este fator também está associado ao aumento no número de segmentos de negócios dentro de uma organização (DOYLE; GE; MCVAY, 2007). Consequentemente, uma maior diversidade de transações comerciais tende a causar menor integração entre os sistemas de controle gerencial, bem como, causar maiores dificuldades de gerenciamento de riscos (GE; MCVAY, 2005).

Para à Bourgeois (1985) e Liebenberg e Hoyt (2003) a complexidade da organização eleva a exposição aos riscos e amplia as dificuldades para uma efetiva estratégia organizacional. Ainda, Beasley, Pagach e Warr (2008) enfatizam que a adoção do ERM depende da complexidade da empresa, sendo que empresas menores e menos complexas tendem a aplicar o ERM de maneira informal e menos estruturada. Gordon, Loeb e Tseng (2009) sugerem uma relação positiva entre a complexidade da empresa e a necessidade de um sistema ERM, sendo que tal relação depende do desempenho da empresa. Assim elaborou-se a terceira hipótese da pesquisa:

$\mathbf{H}_{3}$ : Há relação significativa entre ERMI e a complexidade da empresa para as empresas de alto desempenho empresarial.

Na medida em que o tamanho de uma organização aumenta, maior é a necessidade pela utilização de técnicas de gerenciamento de risco e sendo mais eficazes em toda a empresa. Além disso, organizações maiores podem ter uma capacidade mais elevada de implementar o gerenciamento de risco, haja vista que tendem a possuir mais recursos. Kleffner, Lee e Mcgannon (2003) destacam que as empresas de maior porte são mais suscetíveis à adoção do gerenciamento de risco empresarial.

Percebe-se que a adoção de estratégia de gerenciamento de riscos pode ser influenciada por uma série fatores ede características organizacioanais, sendo a variável tamanho da organização frequentemente utilizada nas pesquisas (KLEFFNER; LEE; MCGANNON, 2003; BEASLEY; PAGACH; WARR, 2008). Colquitt, Hoyt e Lee, (1999) evidenciaram que as características específicas da empresa, como setor e tamanho, afetaram a utilização das técnicas do gerenciamento de risco empresarial.

Estudos demostraram que as grandes empresas possuem maior probabilidade de ter programas de gerenciamento de risco empresarial (COLQUITT; HOYT; LEE, 1999; LIEBENBERG; HOYT, 2003; BEASLEY; CLUNE; HERMANSON, 2005), e ainda, observam uma relação significativa entre o tamanho e a utilização do ERM e o desempenho da empresa (LANG; STULZ, 1994; ALLAYANNIS; WESTON, 2001). Diante destas premissas, elaborou-se a quarta hipótese de pesquisa:

\section{$\mathbf{H}_{4}$ : Há relação significativa entre ERMI e o tamanho da empresa para as empresas de alto desempenho empresarial.}

O conselho de administração tem a responsabilidade pelo gerenciamento dos riscos corporativo, sendo responsável perante os acionistas e outras partes interessadas. Desta maneira, o sucesso da implantação do gerenciamento de riscos tende a aumentar mediante o envolvimento do conselho da administração com o ERM (DICKINSON, 2001).

Constata-se que o ERM é um processo acompanhado pelo conselho de administração e projetado para identificar eventos potenciais que possam afetar a organização. Seu intuito é gerenciar os riscos em consonância com o apetite ao risco desenvolvido, além de fornecer uma garantia razoável quanto à realização dos objetivos organizacionais (COSO, 2004). Ainda referente a gestão de riscos e o conselho de administração, Kleffner, Lee e Mcgannon (2003) e Beasley, Clune e Hermanson, (2005) demostraram que um dos motivos pelos quais as organizações adotam o ERM diz respeito ao incentivo do conselho de administração.

Gordon, Loeb e Tseng (2009) demonstram uma relação positiva entre o monitoramento pelo conselho de administração e o uso de um sistema de ERM, além disso, essa relação será maior para as empresas de maior desempenho. Desta maneira, elaborou-se a quarta hipótese de pesquisa:

$\mathbf{H}_{5}$ : Há uma relação significativa entre ERMI e o monitoramento do conselho de administração para as empresas de alto desempenho empresarial.

O risco da empresa é incorporado dentro da estratégia corporativa de uma organização mediante o contexto do ambiente em que opera (DICKINSON, 2001). As empresas que possuem o gerenciamento de risco empresarial são capazes de compreender melhor os riscos agregados inerentes às diferentes atividades comerciais, proporcionando uma base mais objetiva para a alocação de recursos, melhorando assim a eficiência do capital e o seu desempenho (HOYT; LIEBENBERG, 2011).

Com vistas a explicitar o modelo teórico deste estudo, apresentam-se na Figura 1 as possíveis ligações entre as variáveis analisadas. 
Figura 1: Relação entre o ERM e as variáveis contingenciais frente ao desempenho.

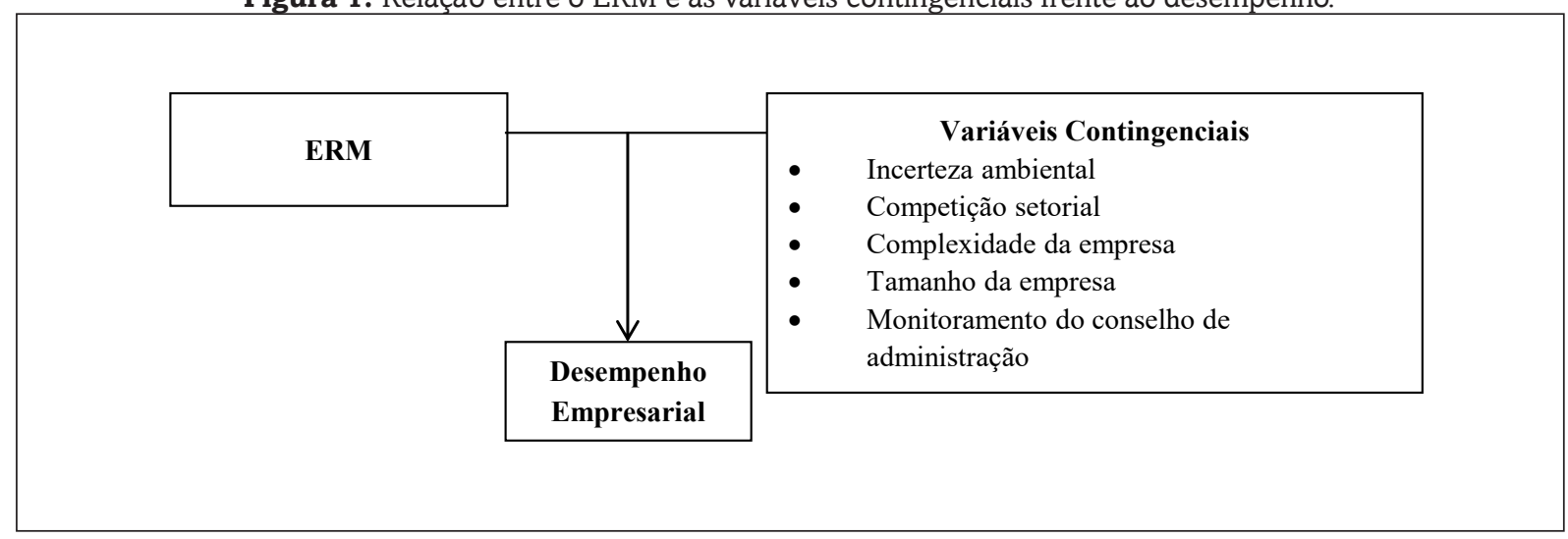

Fonte: Adaptado de Gordon, Loeb e Tseng (2009).

\section{PROCEDIMENTOS METODOLÓGICOS}

Para verificar a relação entre o ERM e as variáveis contingenciais mediante o desempenho empresarial da organização, define-se esta pesquisa como descritiva, documental e quantitativa, sendo que a população do estudo compreende as empresas listadas B3. Inicialmente foram identificadas as empresas cujas informações necessárias para os cálculos do ERMI e os dados relacionados com as variáveis contingenciais estavam disponíveis, com isso o recorte temporal estabelecido para o estudo foi de cinco anos (2012 a 2016), período analisado devido a disponibilidade dos dados. Decorrente dos critérios estabelecidos a amostra é composta por 247, 245, 228, 199 e 187 empresas para os anos de 2012, 2013, 2014, 2015 e 2016, respectivamente, apresentando 1106 observações, sendo 428 empresas classificadas com alto desempenho e 678 observações para as outras empresas.

O Quadro 1 descreve o constructo da pesquisa e contempla as variáveis de análise, a definição constitutiva das variáveis, a fórmula de cálculo aplicada na análise dos dados, a fonte de coleta e estudos correlatos.

Quadro 1 - Variáveis do estudo

\begin{tabular}{|c|c|c|c|c|}
\hline Variáveis & Definição & Fórmula & Coleta & Autores \\
\hline \multicolumn{5}{|c|}{ 1) Variável Dependente (Regressão) } \\
\hline $\begin{array}{c}\text { Enterprise Risk Management } \\
\text { Index } \\
\text { (ERMI) }\end{array}$ & $\begin{array}{l}\text { Utilizado para medir o } \\
\text { gerenciamento de risco } \\
\text { de uma empresa }\end{array}$ & $\begin{array}{c}\text { Equação 1, Equação 2, } \\
\text { Equação 3, Equação 4, } \\
\text { Equação 5, Equação } 6 \text { e } \\
\text { Equação. }\end{array}$ & $\begin{array}{c}\text { Economática }{ }^{\circledR} \\
\text { e } \\
\text { B3 }\end{array}$ & $\begin{array}{c}\text { Gordon, Loeb e Tseng } \\
\text { (2009). }\end{array}$ \\
\hline \multicolumn{5}{|c|}{ 2) Variáveis Independentes (Regressão) } \\
\hline $\begin{array}{l}\text { Incerteza Ambiental } \\
\text { (IA) }\end{array}$ & $\begin{array}{c}\text { Mudança ou variabilidade } \\
\text { no ambiente externo da } \\
\text { organização }\end{array}$ & Equação 8 & & $\begin{array}{c}\text { Duncan (1972); } \\
\text { Milliken (1987); Kren } \\
\text { (1992); Hoque (2004); } \\
\text { Hartmann (2005) }\end{array}$ \\
\hline Concorrência Setorial (CS) & $\begin{array}{l}\text { Proporção das vendas } \\
\text { frente as ao total de } \\
\text { vendas do setor }\end{array}$ & & & $\begin{array}{c}\text { Bourgeois (1985); } \\
\text { Gordon, Loeb e Tseng } \\
\text { (2009). }\end{array}$ \\
\hline $\begin{array}{l}\text { Complexidade da Empresa } \\
\text { (CE) }\end{array}$ & $\begin{array}{l}\text { Diversidade das } \\
\text { transações comerciais }\end{array}$ & $\begin{array}{l}\text { Número de segmentos de } \\
\text { atividade da empresa }\end{array}$ & $\begin{array}{c}\text { Economática }{ }^{\circledR} \\
\text { e }\end{array}$ & $\begin{array}{l}\text { Ge e McVay (2005); } \\
\text { Doyle, Ge e Mcvay, } \\
\text { (2007). }\end{array}$ \\
\hline $\begin{array}{l}\text { Tamanho da empresa } \\
\text { (TAM) }\end{array}$ & Tamanho da organização & Ln do Ativo & B3 & $\begin{array}{l}\text { Francis et al., (2004); } \\
\text { Ge e McVay (2005) }\end{array}$ \\
\hline $\begin{array}{l}\text { Monitoramento do conselho } \\
\text { de adm. (MCA) }\end{array}$ & $\begin{array}{l}\text { Monitoramento } \\
\text { pelo conselho de } \\
\text { administração }\end{array}$ & $\begin{array}{l}\text { Número de diretores } \\
\text { log. de vendas }\end{array}$ & & $\begin{array}{l}\text { Larcker, Richardson e } \\
\text { Tuna (2007) }\end{array}$ \\
\hline
\end{tabular}




\begin{tabular}{|c|c|c|c|c|}
\hline \multicolumn{2}{|c|}{ 3) Variáveis de Desempenho (Utilizadas somente para formação dos Clusters) } \\
\hline ROA & Retorno sobre o Ativo & & Alves e Matias (2014) \\
ROE & Retorno sobre o Patrimônio Líquido & & Economática® & Atz et al. (2017) \\
$\begin{array}{c}\text { Margem } \\
\text { Líquida } \\
\text { (MGL) }\end{array}$ & Margem Líquida & & $\begin{array}{c}\text { Bolton, Chen e Wang } \\
\text { (2011) }\end{array}$ \\
\hline
\end{tabular}

Fonte: Dados da pesquisa, 2018.

Os dados foram obtidos nos Formulários de Referência disponíveis no website da B3 e no banco de dados Economática ${ }^{\circledR}$. Em seguida é demonstrado o cálculo para a variável dependente ERMI, conforme proposto por Gordon, Loeb e Tseng (2009).

\subsection{Enterprise Risk Management Index (ERMI)}

O Índice é baseado nos quatro indicadores do ERM do COSO, conforme (GORDON; LOEB; TSENG,2009). Desta maneira, a eficácia do índice de ERM em uma organização é denominado ERMI e decorre da capacidade da empresa em atingir os seguintes objetivos: i) estratégia; ii) operações; iii) relatórios; iv) conformidade. O ERMI é então construído por estes quatro indicadores estabelecidos, como se demonstra na Eq. (1):

$$
\text { ERMI }=\sum_{k=1}^{1} \text { Estratégia }+\sum_{k=1}^{1} \text { Operação }+\sum_{k=1}^{1} \text { Relatório }+\sum_{k=1}^{1} \text { Conformidade }
$$

A definição e os dados relacionados para cada indicador são explicados a seguir.

\subsubsection{Estratégia}

O indicador Estratégia se relaciona com a maneira como uma empresa se posiciona no mercado em relação à sua concorrência. Ao executar sua estratégia, uma empresa tenta desenvolver uma vantagem competitiva em relação aos participantes da mesma indústria (PORTER, 2008). Essa vantagem competitiva deve reduzir o risco geral de falha de uma empresa e, assim, aumentar seu desempenho. Assim, a exemplo do estudo de Gordon, Loeb e Tseng (2009).), a métrica adotada para estabelecer se uma empresa tem ou não uma estratégia bem sucedida é a quantidade de desvios padrão que suas vendas (Economática $\left.{ }^{\circledR}\right)$ se desviam das vendas de seu setor, conforme demonstrado abaixo:

$$
\text { Estratégia }=\frac{\text { vendas }-\mu_{\text {vendas }}}{\sigma_{\text {vendas }}}
$$

Onde:

Vendas = Vendas da empresa;

$\mu_{\text {vendas }}=$ Vendas médias da empresa;

$\sigma_{\text {vendas }}=$ Desvio padrão de vendas de todas as empresas.

\subsubsection{Operação}

Por sua vez, a Operação é estabelecida com base na relação entrada-saída no processo das operações de uma empresa (Banker et al., 1989). Uma maior eficiência operacional deve reduzir o risco geral de falha e assim aumentar o desempenho e valor da empresa. Portanto, o volume de negócios, definido como vendas (Economática ${ }^{\circledR}$ ), dividido pelos ativos totais (Economática $\left.{ }^{\circledR}\right)$ é uma medida de eficiência operacional (Kiymaz, 2006). Esta medida é mensurada da seguinte maneira:

$$
\text { Operação }=\frac{\text { Vendas }}{\text { Total de ativos }}
$$




\subsubsection{Relatório}

Para o modelo, segundo Gordon, Loeb e Tseng (2009) um relatório financeiro inadequado deve aumentar o risco geral de falha de uma empresa e assim diminuir seu desempenho e valor. Uma medida da confiabilidade de relatórios de uma empresa, adotada por estudos como qualidade da informação evidenciada nos relatórios contábeis, é a proporção relativa do valor absoluto dos accruals normais divididos pela soma do valor absoluto dos accruals normais e anormais. Os valores absolutos são usados porque os acúmulos normais e os acúmulos anormais podem ser negativos. Os accruals anormais são estimados usando o modelo Jones Modificado. Desta maneira, a variável relatório será medido, conforme equação abaixo:

$$
\text { Relatório }=\frac{(\text { AccrualsNormal })}{(\text { AccrualsNormal })+(\text { AccrualsAnormal })}
$$

\section{Accruals Normais}

Segundo Hribar e Collins (2002), os accruals normais (AT) podem ser identificados por dois enfoques: pelo Balanço e pela Demonstração do Fluxo de Caixa (DFC). No entanto, para o caso brasileiro, o enfoque do Balanço demonstra-se mais adequado, uma vez que a DFC não é uma demonstração financeira exigida. No enfoque do balanço os AT são encontrados pela seguinte especificação:

$$
A T=(\triangle A C-\Delta C x)-(\Delta P C-\Delta D i v)-D e p
$$

Onde:

$\Delta=$ Representa a variação anual observada na variável;

$\mathrm{AC}=$ Ativo Circulante

$\mathrm{Cx}=$ Caixa e equivalentes a caixa;

$\mathrm{PC}=$ Passivo Circulante;

Dív = Dívida de curto prazo e

Dep = Despesa com depreciação e amortização.

\section{Accruals Anormais}

O Modelo Modificado de Jones é formulado com o intuito de eliminar a tendência conjeturada do Modelo de Jones para mensurar accruals discricionários com erro quando a discricionariedade sobre as receitas é exercida. No Modelo Modificado, os accruals não discricionários são estimados como:

$$
A C T=\alpha_{1}\left(\frac{1}{A_{t-1}}\right)+\alpha_{2}\left(\Delta R E V_{t}-\Delta R E C E C_{i t}\right)+\alpha_{3}\left(P P E_{t}\right)+\varepsilon_{i t}
$$

Onde:

ACT $=$ Accruals totais;

$\mathrm{A}_{\mathrm{it}-1}=$ ativo total da firma i no ano $\mathrm{t}-1$;

$\Delta \mathrm{REV}_{\mathrm{it}}=$ variação receita bruta da firma i entre os anos $\mathrm{t}$ e $\mathrm{t}-1$, ponderados pelos ativos totais no final do período $\mathrm{t}-1$;

$\triangle \mathrm{REC}_{\mathrm{it}}=$ variação das contas a receber (clientes) da firma i entre os anos t e t-1, ponderados pelos ativos totais no final do período $\mathrm{t}-1$; $\mathrm{PPE}_{\mathrm{it}}=$ imobilizado da firma i no ano $\mathrm{t}$, ponderados pelos ativos totais no final do período $\mathrm{t}-1$;

$\varepsilon_{\text {it }}=$ resíduo da regressão para a firma i no ano t.

As estimativas de $\alpha 1$, $\alpha 2$ e $\alpha 3$ e accruals não discricionários são aquelas obtidas do modelo original de Jones (1991). Para Dechow et al. (1995), o modelo original de Jones é ajustado para a evidenciar as mudanças nos recebíveis. Desta maneira, demostrando implicitamente que todas as mudanças que ocorrem nos créditos de vendas no período resultam do gerenciamento de resultados. Tal fato acontece, devido ao fato que gerenciar resultados por exercício da discricionariedade sobre o reconhecimento de receitas sobre crédito de vendas é mais fácil do que gerenciar resultado pelo exercício de discricionariedade sobre o reconhecimento de receitas sobre caixa (MARTINEZ, 2007).

\subsubsection{Conformidade}

Diz respeito à conformidade com as leis e regulamentos. Quando uma empresa promove uma adequada gestão de compliance, autores como Shavell (1982) e Gordon, Loeb e Tseng (2009), consideram que deve haver uma diminuição do risco geral de falha de uma empresa e, por conseguinte, aumentar seu desempenho e valor. A medida da conformidade utilizada no estudo, similar ao adotado no estudo de Gordon, Loeb e Tseng (2009), é a proporção de honorários do auditor para a receita líquida de vendas $\left(\right.$ Economática $\left.^{\circledR}\right)$. 


$$
\text { Conformidade }=\frac{\text { Honorários Auditor }}{\text { Total de Ativos }}
$$

Em seguida, demonstra-se o cálculo da variável independente Incerteza.

\subsection{Incerteza Ambiental (IA)}

Com base em características delineadas por Duncan (1972), a incerteza ambiental é utilizada como variável na construção de diversos estudos na literatura de contabilidade gerencial como Milliken (1987), Hoque (2004), Hartmann (2005). Neste estudo IA é medida por meio da combinação das três seguintes métricas (KREN, 1992):

(1) Mercado: Coeficiente de variação de vendas;

(2) Tecnológico: Coeficiente de variação da soma de P\&D e despesas de capital dividido pelo total de ativos; e

(3) Renda: Coeficiente de variação do lucro líquido antes de impostos.

Para cada empresa, o coeficiente de variação é calculado no período 2012-2016 com base nas primeiras diferenças. A medida composta de IA e os coeficientes individuais são calculados, conforme o modelo de Gordon, Loeb e Tseng (2009), como demonstrado na equação (4) abaixo:

$$
I A=\log +\left(\sum_{k=1}^{3} C V\left(X_{k}\right)\right)
$$

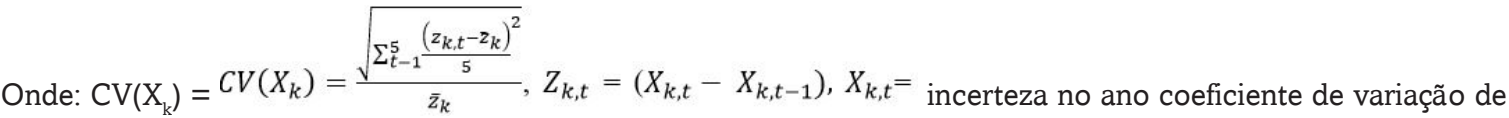
incerteza para representar os anos 2012-2016, para representar o mercado, incerteza tecnológica ou de renda, significa mudanças em 5 anos de incerteza. O valor absoluto de é usado como o denominador de para evitar o caso em que um negativo transforma uma situação de incerteza em uma situação de certeza.

\subsection{Teste do modelo}

Inicialmente, utilizou-se a técnica de Cluster Analysis para classificar as organizações em grupos de desempenho empresarial. Clusters ou análise de conglomerados foi utilizada neste trabalho para classificar as empresas em grupos homogêneos, conforme os elementos que influenciam o desempenho empresarial das organizações por meio da aplicação do vizinho mais próximo. Segundo Marôco (2011), Cluster é uma técnica exploratória que utiliza a análise multivariada, da qual agrupar os sujeitos ou variáveis em grupos homogêneos ou compactos relativamente a uma ou características comuns. Ainda, para o autor os agrupamentos dos sujeitos ou variáveis a partir de medidas de semelhanças ou de distâncias entre dois Cluster de observações, com a utilização de técnicas hierárquicas ou não-hierárquica de agrupamento.

Em seguida, testou-se a relação entre a gestão de risco empresarial e as variáveis contingenciais, considerando que as empresas do grupo com melhor desempenho empresarial tendem à uma melhor correspondência adequada entre o gerenciamento de risco empresarial e as variáveis incerteza ambiental, concorrência setorial, complexidade da empresa, tamanho e monitoramento do conselho de administração, conforme Gordon, Loeb e Tseng (2009).

Assim, verificou-se a relação entre o ERMI (que é usado como proxy para o gerenciamento de riscos empresariais conforme Equação 1) e os fatores de contingência. Realizou-se a regressão OLS (Ordinary Least Squares), robusta e controlando setor e ano, utilizando-se o software Statistics Data Analysis (Stata® 13.0).

$$
\begin{gathered}
\text { ERMI }=\beta_{0}+\beta_{1} I A+\beta_{2} C S+\beta_{3} C E+\beta_{4} T A M+\beta_{5} M C A+\text { Efeitor fixos ano }+ \\
\text { Efeitos fixos setor }+\varepsilon
\end{gathered}
$$

Onde:

ERMI = Enterprise Risk Management Index;

IA = Incerteza Ambiental;

$\mathrm{CS}=$ Concorrência Setorial;

$\mathrm{CE}=$ Complexidade da Empresa;

TAM = Tamanho da empresa;

MCA = Monitoramento do Conselho de Administração;

$\varepsilon=$ Erro da regressão. 
O modelo proposto enfatiza a perspectiva holística sobre como todas as variáveis de contingência estão relacionadas ao gerenciamento de risco empresarial. As empresas que buscam monitorar tais contingências, provavelmente terão um desempenho maior que aqueles que seguem um modelo diferente (GORDON; LOEB; TSENG, 2009),).

\section{APRESENTAÇÃO E ANÁLISE DOS RESULTADOS}

Nesta seção realiza-se a descrição e análise dos resultados pertinentes ao presente estudo, conforme a Tabela 1.

Tabela 1 - Estatística descritiva das variáveis

\begin{tabular}{|c|c|c|c|c|c|c|}
\hline \multirow{2}{*}{ Variáveis } & \multicolumn{3}{|c|}{ Alto Desempenho } & \multicolumn{3}{|c|}{ Outras empresas } \\
\hline & Média & Med. & D.P & Média & Med. & D.P \\
\hline ERMI & 0,9820 & 0,7854 & 1,1361 & 0,9230 & 0,716 & 1,0824 \\
\hline IA & 5,4859 & 5,6509 & 0,9319 & 3,8966 & 4,5664 & 2,7157 \\
\hline CS & 0,3029 & 0,0812 & 0,3784 & 0,2874 & 0,0644 & 0,3654 \\
\hline CE & 2,8014 & 3,00 & 1,0152 & 2,7734 & 3 & 0,7430 \\
\hline TAM & 14,7700 & 15,0416 & 1,9817 & 14,3025 & 14,5088 & 1,9827 \\
\hline MCA & 1,9626 & 1,8790 & 1,6436 & 1,7100 & 1,8065 & 1,4298 \\
\hline
\end{tabular}

Legenda: ERMI: Enterprise Risk Management Index. IA: Incerteza Ambiental. CS: Concorrência Setorial. CE: Complexidade da Empresa. TAM: Tamanho da empresa. MCA: Monitoramento do Conselho de Administração. Fonte: Dados da pesquisa, 2018

Nota-se uma diminuição dos indicadores que mensuram as variáveis contingenciais para as empresas classificadas como outras empresas. O que demonstra que os indicadores contingenciais são maiores paras as empresas com alto desempenho, sendo assim estas empresas estão mais propensas a impactos contingenciais na realização de suas atividades.

O ERMI, que e demostra a capacidade da empresa em atingir os seguintes objetivos em estratégia, operações, relatórios e conformidade, é mais elevado nas empresas com maior desempenho. Além disso, constata-se que em empresas com alto desempenho o gerenciamento de riscos corporativos tende a ser maior. Este resultado corrobora os achados de Gordon, Loeb e Tseng (2009).

Em seguida, na Tabela 3 demonstra-se a Correlação de Pearson entre as variáveis analisadas no estudo.

Tabela 2 - Correlação de Pearson entre as variáveis

\begin{tabular}{c|c|c|c|c|c|c}
\hline VARIÁVEL & ERMI & IA & CS & CE & TAM & MCA \\
\hline ERMI & 1 & 0.1593 & 0.3213 & $0.0223^{\star \star}$ & $0.0463^{\star \star}$ & $0.0131^{\star \star}$ \\
IA & & 1 & 0.1510 & $-0.0913^{\star}$ & 0.4082 & $0.0772^{\star}$ \\
CS & & & 1 & 0.1504 & 0.1186 & $0.0616^{\star \star}$ \\
CE & & & & 1 & -0.1420 & $-0.0477^{\star \star}$ \\
TAM & & & & & & 0.2457 \\
MCA & & & & & & \\
\hline
\end{tabular}

Legenda: ERMI: Enterprise Risk Management Index. IA: Incerteza Ambiental. CS: Concorrência Setorial. CE: Complexidade da Empresa. TAM: Tamanho da empresa. MCA: Monitoramento do Conselho de Administração.

Notas: Níveis de significância: ${ }^{*} p<0,1,{ }^{* *} p<0,05,{ }^{* \star *} p<0,01$. Fonte: Dados da pesquisa, 2018.

Referente à Correlação de Pearson entre as variáveis demonstrado na Tabela 2, percebe-se a existência da correlação entre as variáveis. O MCA apresenta uma correlação positiva com o IA, CS, e negativa com CE. Além disso, observa-se uma correlação negativa do CE com IA.

No que se que refere ao ERMI percebe-se uma correlação positiva com CE, TAM e MCA, deste modo, o índice de gestão de risco é correlacionado com os índices contingenciais.

De modo geral, os dados da Tabela 2 demonstram que não há correlação elevada entre as variáveis analisadas, o que permite descartar possíveis problemas de multicolinearidade nos modelos de regressão a seguir.

Em seguida realizou-se Cluster Analysis, agrupando as organizações com desempenhos semelhantes por meio do pacote estatístico SPSS em sua versão 22. O procedimento que determinou as similaridades da amostra foi o método de ligação simples, aplicando-se a distância mínima e métrica euclidiana. Por fim utilizou-se o número final de grupos que 
resultou na utilização de dois Clusters. No que tange, à interpretação e perfil dos conglomerados, na Tabela 3 apresentam-se a quantidade de empresas nos conglomerados (cluster) representados pelos Cluster 1 e Cluster 2

Tabela 3 - Composição de empresas por conglomerados

\begin{tabular}{l|l|l|l|l|l}
\hline Cluster & $\mathbf{2 0 1 2}$ & $\mathbf{2 0 1 3}$ & $\mathbf{2 0 1 4}$ & $\mathbf{2 0 1 5}$ & 2016 \\
\hline Cluster 1 (Alto desempenho) & 82 & 83 & 102 & 86 & 75 \\
Cluster 2 (Outras empresas) & 165 & 162 & 126 & 113 & 112 \\
Total & $\mathbf{2 4 7}$ & $\mathbf{2 4 5}$ & $\mathbf{2 2 8}$ & $\mathbf{1 9 9}$ & $\mathbf{1 8 7}$ \\
\hline
\end{tabular}

Fonte: Dados da pesquisa, 2018.

Verificou-se uma maior concentração de empresas, no período em análise, no Cluster 2, no entanto, constata-se uma variação de quantidades de empresas nos anos analisados. Por sua vez, os resultados da regressão, no que se refere ao modelo desenvolvido, são evidenciados na Tabela 4. A tabela contempla os dois modelos de regressão de alto desempenho e outras empresas, conforme aplicado pelo método de Cluster com os indicadores de desempenho. Espera-se evidenciar uma maior relação entre ERMI e as variáveis contingenciais no modelo de alto desempenho.

Tabela 4 - Relação entre o ERMI e as variáveis contingenciais

\begin{tabular}{|c|c|c|c|c|c|c|}
\hline \multirow{2}{*}{ Variáveis } & \multicolumn{3}{|c|}{ Modelo Alto Desempenho } & \multicolumn{3}{|c|}{ Modelo Outras Empresas } \\
\hline & Coef. & Teste $\mathbf{t}$ & Sig. & Coef. & Teste $t$ & Sig. \\
\hline _Cons & 1.0210 & 1.92 & 0.056 & 1.0071 & 1.93 & 0.054 \\
\hline IA & 0.7076 & 4.56 & 0.000 & 0.5676 & 4.64 & 0.000 \\
\hline CS & 0.5284 & 3.93 & 0.000 & 0.8840 & 9.07 & 0.000 \\
\hline $\mathrm{CE}$ & -0.0355 & -0.62 & 0.537 & -0.0620 & -0.97 & 0.334 \\
\hline TAM & -0.2776 & -3.80 & 0.000 & -0.2256 & -3.36 & 0.001 \\
\hline MCA & 0.0310 & 0.93 & 0.351 & -0.0011 & -0.04 & -0.04 \\
\hline Efeitos Fixos Setor e Ano & \multicolumn{3}{|c|}{ Sim } & \multicolumn{3}{|c|}{ Sim } \\
\hline $\mathrm{R}^{2}$ & \multicolumn{3}{|c|}{18,93} & \multicolumn{3}{|c|}{18,17} \\
\hline Sig. Modelo & \multicolumn{3}{|c|}{0,000 * } & \multicolumn{3}{|c|}{$0,000 *$} \\
\hline Durbin-Watson & \multicolumn{3}{|c|}{2,056} & \multicolumn{3}{|c|}{2,19} \\
\hline Shapiro-Wilk & \multicolumn{3}{|c|}{0,000} & \multicolumn{3}{|c|}{0,000} \\
\hline $\mathrm{N}^{\circ}$ Observações & \multicolumn{3}{|c|}{428} & \multicolumn{3}{|c|}{678} \\
\hline VIF & \multicolumn{3}{|c|}{$1,13-5,25$} & \multicolumn{3}{|c|}{$1,07-3,97$} \\
\hline
\end{tabular}

* Significância ao nível de $1 \%$.

** Significância ao nível de 10

Fonte: Dados da pesquisa, 2018.

Na Tabela 2, verifica-se que o Durbin-Watson apresentou valor acima de 2, demonstrando que a independência dos erros nos dados analisados é satisfatória e que não existe autocorrelação entre os resíduos (MARÔCO, 2011). Posteriormente, realizou-se o teste de Shapiro-Wilk para verificar a normalidade dos dados, percebe-se que a distribuição dos resíduos não é normal, pois houve significância ao nível de 1\%. Por fim, o teste de multicolinearidade (VIF) demonstra a inexistência de problemas de multicolinearidade, tendo em vista que seus valores devem estar entre 1 e 10 (HAIR JR et al., 2009). Desta maneira, percebe-se que não houve problemas de multicolinearidade, visto que os valores das variáveis analisadas ficaram entre 1,07 a 5,25 nos modelos analisados.

Contata-se que ambos os modelos são estatisticamente significantes, sendo que o poder explicativo (R2) foi de 18,93\% para o modelo de alto desempenho e 18,17\% para o modelo de baixo desempenho. Ao analisar-se a Equação 3 (que trata do ERMI frente aos fatores contingenciais), no modelo de alto desempenho, observa-se que há uma persistência nos resultados no que tange os coeficientes $\alpha 2$ e $\alpha 3$, sendo estes significativos ao nível de $1 \%$. As organizações do modelo de alto desempenho demonstraram uma melhor correspondência entre o ERMI e as variáveis de Incerteza 
Ambiental (IA) e Concorrência Setorial (CS). Estes resultados sugerem que as empresas categorizadas como de alto desempenho estão dando maior importância as informações obtidas por meio dos dados de IA, constituída por variáveis de estratégia, operação, relatório e conformidade, logo, a eficácia de seu ERMI tende a ser superior.

De mesmo modo, a maior observância da Concorrência Setorial parece estar atrelada a um alto índice de ERMI. Estes resultados corroboram com os achados de Gordon, Loeb e Tseng (2009), ampliando ainda mais sua robustez, tendo em vista que os autores analisaram tais correlações apenas no ano de 2005, no contexto de empresas americanas, sendo que no presente modelo observam-se o espaço temporal de 5 anos (2012 - 2016). Todavia, diferentemente dos resultados de Gordon et al. (2009), não foram encontrados resultados significativos para as variáveis de Complexidade Empresarial (CE), e Monitoramento do Conselho de Administração (MCA).

Assim, confirma-se a hipóteses $\mathbf{H}_{\mathbf{1}}$ : Há relação significativa entre ERMI e incerteza ambiental para as empresas de alto desempenho empresarial. Constata-se, a partir desses achados, que o ERMI possui relação com as incertezas ambientais sofridas pelas organizações quando elas possuem um alto desempenho. Destaca-se que a hipótese nula foi rejeitada, o que confirma a relação significativa entre ERMI e IA.

Segundo Arena et al. (2010) destacam que o ERM pode ser visto como uma maneira de os gestores prepararem suas organizações frente as incertezas existentes no âmbito corporativo. Além disso, Gordon, Loeb e Tseng (2009), evidenciaram que o ERM destina-se a identificar e gerenciar eventos futuros incertos que podem influenciar negativamente o desempenho da organização. Na sequência frente aos achados do estudo, confirma-se a hipótese $\mathbf{H}_{2}$ : Há relação significativa entre ERMI e a concorrência setorial para as empresas de alto desempenho empresarial. O que indica que o ERMI possui relação com a concorrência setorial nas organizações que possuem um alto desempenho. Os achados de Khandwalla (1972) demostram que a concorrência setorial pode influenciar os controles de gestão. Além disso, Meulbroek (2002) revelou que o aumento da concorrência influencia a ênfase do gerenciamento de riscos nas organizações. Para Gordon, Loeb e Tseng (2009), quanto maior o nível de concorrência para as vendas de uma organização, mais competente deve ser o seu sistema de ERM, assim como, maior deve ser a adequação de ERM frente a concorrência setorial o que também é confirmado nesta pesquisa.

Em seguida, confirma-se a hipótese $\mathbf{H}_{4}$ : Há relação significativa entre ERMI e o tamanho da empresa para as empresas de alto desempenho empresarial. Constata-se que o ERMI possui relação com a variável tamanho da empresa no grupo de alto desempenho. Kleffner, Lee e Mcgannon (2003) evidenciaram que as empresas de maior porte possuem mais probabilidade de adotar gerenciamento de risco devido à necessidade de uma estratégia abrangente de gestão de riscos. Colquitt, Hoyt e Lee (1999) evidenciaram que as características específicas da empresa, como setor e tamanho, afetaram a utilização das técnicas do gerenciamento de risco empresarial.

Todavia as hipóteses $\mathbf{H}_{3}$ e $\mathbf{H}_{5}$, pertinentes respectivamente a relação positiva e significativa entre ERMI e Complexidade da Empresa (CE) e Monitoramento do Conselho de Administração (MCA) não foram suportadas. Referente à complexidade da empresa este estudo diverge de Bourgeois (1985) e Liebenberg e Hoyt (2003), Beasley, Pagach e Warr (2008) que demostram que complexidade da organização amplia os riscos e dificulta a consecução da estratégia organizacional.

No que tange o monitoramento do conselho de administração percebe-se que o ERMI não possui relação com este indicador, no entanto, diferentemente do esperado, apresenta uma relação positiva com as empresas que não estão no conglomerado de alto desempenho. O resultado pode indicar que o Conselho de Administração tenderia a centrar maior atenção ao ERM quando a empresa está com seu desempenho inferior ao observado por seus concorrentes diretos. Para Dickinson (2001), o conselho de administração tem a responsabilidade pelo risco corporativo da empresa. Kleffner, Lee e Mcgannon (2003) e Beasley, Clune e Hermanson (2005) demostraram que os motivos para a adoção do ERM nas organizações é a influência do conselho de administração para a sua implantação, no entanto, contrariando as expectativas, no presente estudo tal evidência é constatada quando as empresas não possuem alto desempenho.

Em um segundo momento, ao analisar-se o ERMI e os fatores contingenciais (Equação 3), no modelo para outras empresas, ou seja, aquelas que não se enquadram no grupo de alto desempenho, observa-se que somente a variável Tamanho da empresa (TAM) é significativa ao nível de 1\%. Todavia, observa-se que tais resultados não são sustentados pelas demais variáveis e ainda, divergem dos resultados observados na literatura como Gordon, Loeb e Tseng (2009).

\section{CONSIDERAÇÕES FINAIS}

Sendo a gestão de risco uma preocupação fundamental das organizações, frente a um âmbito empresarial cada vez mais global e dinâmico, diversas abordagens vêm sendo observadas no que tange o gerenciamento de riscos, principalmente no que se refere ao seu tratamento de um modo mais abrangente, com uma visão holística da gestão de riscos. Nesta perspectiva, o estudo proposto objetivou verificar a relação entre o ERM das organizações e as variáveis contingenciais mediante o desempenho empresarial da organização. Tendo como base as evidências verificadas na literatura e os resultados dos diversos testes estatísticos, pode-se realizar algumas inferências com relação ao fenômeno analisado.

O ERM busca dirimir os riscos organizacionais, e por sua vez, maximizar os resultados da organização, elevando assim o valor da empresa. Uma vez que os fatores contextuais são geralmente variáveis exógenas (GORDON, LOEB e TSENG, 2009), os resultados do estudo sugerem que empresas de alto desempenho estão preocupando-se e atribuin- 
do maior importância as variáveis contingenciais do que as demais organizações, quando da implementação do ERM, haja vista a confirmação das hipóteses $\mathbf{H}_{1}$ para incerteza ambiental (IA), $\mathbf{H}_{2}$ referente à Concorrência Setorial (CS) e $\mathbf{H}_{4}$ para Tamanho da Empresa (TAM).

A incerteza ambiental gera dificuldades para empresas no que tange a previsão dos eventos futuros que possam afetar suas operações. Os riscos associados a uma resposta apropriada aos eventos de incerteza ambiental sugerem que as organizações tendem a monitorar a incerteza ambiental com intuito de não sofrer impactos negativos em seus resultados, logo, empresas com um sistema de ERM eficiente (alto ERMI) estão propensas a um maior monitoramento das incertezas ambientais. No mesmo sentido, a concorrência setorial deve ser monitorada com atenção pelas organizações. Analisar os produtos e serviços oferecidos no mercado pelas demais empresas, tanto similares como distintos, pode auxiliar a entidade na competição por vendas nos diversos nichos de mercado. Assim, empresas com maior gerenciamento destas informações, tendem a possuir maiores índices de ERMI. Infere-se ainda, que organizações de tamanho elevado, ou seja, aquelas consideradas de maior porte, tendem a adotar sistemas de gerenciamento de risco.

Corroborando com os achados de Baxter et al. (2013), a qualidade do gerenciamento de risco empresarial, demonstra-se associada ao desempenho organizacional. Todavia alguns resultados possuem fragilidades, tendo em vista que as variáveis contingenciais como Complexidade Empresarial (CE) e Monitoramento do Conselho de Administração (MCA) não se demonstraram significantes no modelo, o que levou respectivamente a não confirmação das hipóteses $\mathbf{H}_{3}$ e $\mathbf{H}_{5}$.

No entanto, destaca-se que diferentemente de estudos anteriores como Beasley, Clune e Hermanson (2005), nos modelos testados nesta pesquisa, evidenciou-se uma maior influência do conselho de administração no sentido de instigar a adoção de sistemas de ERM nas organizações que não pertencem Cluster de alto desempenho, do que naquelas pertencem. Por fim, conclui-se por meio dos resultados obtidos com o modelo estatístico que empresas de alto desempenho atribuem maior importância às variáveis contingenciais do que as demais organizações, quando da implementação do ERM.

Algumas limitações podem ser identificadas no presente estudo no que tange seus resultados, devido aos mesmos serem específicos para amostra utilizada, todavia, com o diferencial de ter-se observado o período temporal de 5 anos (2012 - 2016). Recomenda-se para pesquisas futuras a investigação de outras amostras, possivelmente de países distintos, sendo realizada uma análise comparativa entre os diferentes contextos. Outra possibilidade seria a realização de pesquisa análoga, utilizando-se outras variáveis contingenciais e/ou adicionando tais variáveis na presente análise. No mesmo sentido, uma segregação da amostra sendo realizada uma análise comparativa entre setores poderia originar interessantes inferências relacionadas ao fenômeno em análise.

\section{REFERÊNCIAS}

ATZ, Fernadno; GERHARD, Melissa; FREITAG, Viviane da Costa; VANTI, Adolfo Alberto; COBO, Angel. Análise do relacionamento existente entre a governança da tecnologia da informação, o gerenciamento do risco corporativo e as funções de compliance. São Paulo: SEMEAd. 2017.

ALVES, Maria Teresa; MATIAS, Francisco José Ferreira. Gestão e divulgação do risco operacional e os acordos de basileia: o caso dos maiores bancos portugueses. Revista Universo Contábil, v. 10, n. 3, p. 172-193, 2014.

ARENA, Marika; ARNABOLDI, Michela; AZZONE, Giovanni. The organizational dynamics of enterprise risk management. Accounting, Organizations and Society, v. 35, n. 7, p. 659-675, 2010.

BAXTER, Ryan; BEDARD, Jean; HOITASH, Rani; YEZEGEL, Ari. Enterprise risk management program quality: Determinants, value relevance, and the financial crisis. Contemporary Accounting Research, v. 30, n. 4, p. 1264-1295, 2013.

BEASLEY, Mark; CLUNE, Richard; HERMANSON, Dana. Enterprise risk management: An empirical analysis of factors associated with the extent of implementation. Journal of accounting and public policy, v. 24, n. 6, p. 521-531, 2005.

BEASLEY, Mark; PAGACH, Don; WARR, Richard. Information conveyed in hiring announcements of senior executives overseeing enterprise-wide risk management processes. Journal of Accounting, Auditing \& Finance, v. 23, n. 3, p. 311-332, 2008.

BOLTON, Patrick; CHEN, Hui; WANG, Neng. A unified theory of Tobin's q, corporate investment, financing, and risk management. The journal of Finance, v. 66, n. 5, p. 1545-1578, 2011

BOURGEOIS III, Jay. Strategic goals, perceived uncertainty, and economic performance in volatile environments. Academy of management journal, v. 28, n. 3, p. 548-573, 1985.

BROMILEY, Philip; MCSHANE, Michael; NAIR, Anil; RUSTAMBEKOV, Elzotbek. Enterprise risk management: Review, critique, and research directions. Long range planning, v. 48, n. 4, p. 265-276, 2015.

CHENHALL, Robert H; MORRIS, Deigan. The impact of structure, environment, and interdependence on the perceived usefulness of management accounting systems. Accounting Review, p. 16-35, 1986.

COHEN, Jeffrey; KRISHNAMOORTHY, Ganesh; WRIGHT, Arnold. Enterprise Risk Management and the Financial Reporting Process: The Experiences of Audit Committee Members, CFO s, and External Auditors. Contemporary Accounting Research, v. 34, n. 2, p. 1178-1209, 2017. 
COLQUITT, L. Lee; HOYT, Robert; LEE, Ryan. Integrated risk management and the role of the risk manager. Risk Management and Insurance Review, v. 2, n. 3, p. 43-61, 1999.

COSO. Enterprise risk management - Integrated framework. New York, NY: Committee of Sponsoring Organizations of the Treadway Commission. 2004

DECHOW, Patricia M.; SLOAN, Richard G.; SWEENEY, Amy P. Detecting earnings management. Accounting review, p. 193-225, 1995.

DICKINSON, Gerry. Enterprise risk management: Its origins and conceptual foundation. The Geneva Papers on Risk and Insurance. Issues and Practice, v. 26, n. 3, p. 360-366, 2001.

DOYLE, Jeffrey; GE, Weili; MCVAY, Sarah. Determinants of weaknesses in internal control over financial reporting. Journal of accounting and Economics, v. 44, n. 1-2, p. 193-223, 2007.

DUNCAN, Robert. Characteristics of organizational environments and perceived environmental uncertainty. Administrative science quarterly, p. 313-327, 1972

FRANCIS, Jennifer; RYAN, LaFond; PER Olsson; SCHIPPER, Katherine. Costs of equity and earnings attributes. The accounting review, v. 79, n. 4, p. 967-1010, 2004.

GATZERT, Nadine; MARTIN, Michael. Determinants and value of enterprise risk management: empirical evidence from the literature. Risk Management and Insurance Review, v. 18, n. 1, p. 29-53, 2015.

GE, Weili; MCVAY, Sarah. The disclosure of material weaknesses in internal control after the Sarbanes-Oxley Act. Accounting Horizons, v. 19, n. 3, p. 137-158, 2005.

GORDON, Lawrence A; LOEB, Martin P; TSENG, Chih-Yang. Enterprise risk management and firm performance: A contingency perspective. Journal of Accounting and Public Policy, v. 28, n. 4, p. 301-327, 2009.

GUJARATI, Damodar; PORTER, Dawn. Econometria Básica-5. Amgh Editora, 2011.

HAIR, Joseph; BLACK, William; BABIN, Barry; ANDERSON, Rolph; TATHAM, Ronald. Análise multivariada de dados. Bookman Editora, 2009.

HARTMANN, Frank. The effects of tolerance for ambiguity and uncertainty on the appropriateness of accounting performance measures. Abacus, v. 41, n. 3, p. 241-264, 2005.

HOQUE, Zahirul. A contingency model of the association between strategy, environmental uncertainty and performance measurement: impact on organizational performance. International Business Review, v. 13, n. 4, p. 485-502, 2004.

HOYT, Robert; LIEBENBERG, Andre. The value of enterprise risk management. Journal of risk and insurance, v. 78, n. 4, p. 795-822, 2011.

KHANDWALLA, Pradip. Environment and its impact on the organization. International studies of management \& organization, v. 2, n. 3, p. 297-313, 1972.

KLEFFNER, Anne; LEE, Ryan; MCGANNON, Bill. The effect of corporate governance on the use of enterprise risk management: Evidence from Canada. Risk Management and Insurance Review, v. 6, n. 1, p. 53-73, 2003.

KREN, Leslie. Budgetary participation and managerial performance: The impact of information and environmental volatility. Accounting Review, p. 511-526, 1992.

LAWRENCE, Paul; LORSCH, Jay. Differentiation and integration in complex organizations. Administrative science quarterly, p. 1-47, 1967.

LARCKER, David F; RICHARDSON, Scott; TUNA, Irem. Corporate governance, accounting outcomes, and organizational performance. The accounting review, v. 82, n. 4, p. 963-1008, 2007.

LIEBENBERG, Andre; HOYT, Robert. The determinants of enterprise risk management: Evidence from the appointment of chief risk officers. Risk Management and Insurance Review, v. 6, n. 1, p. 37-52, 2003.

MARÔCO, João. Análise Fatorial com o SPSS Statistics. Pêro Pinheiro: ReportNumber-Análise e, 2011.

MARTINEZ, Antônio Lopo. Detectando earnings management no Brasil: estimando os accruals discricionários. Revista Contabilidade \& Finanças, v. 19, n. 46, p. 7-17, 2008.

MIKES, Anette; KAPLAN, Robert. Towards a contingency theory of enterprise risk management. American Accounting Association - Management Accounting Section (MAS), Meeting Paper, 2013.

MILLIKEN, Frances. Three types of perceived uncertainty about the environment: State, effect, and response uncertainty. Academy of Management review, v. 12, n. 1, p. 133-143, 1987.

MEULBROEK, Lisa. A senior manager's guide to integrated risk management. Journal of Applied Corporate Finance, v. 14, n. 4, p. 56-70, 2002.

NOCCO, Brian; STULZ, René. Enterprise risk management: Theory and practice. Journal of applied corporate finance, v. 18, n. 4, p. 8-20, 2006.

PAGACH, Donald; WARR, Richard. The effects of enterprise risk management on firm performance. Social Science Research Network (SSRN), 2010. 
PELEIAS, Ivam Ricardo; CAETANO, Gilberto; PARISI, Claudio; PEREIRA, Anísio Candido. Produção científica sobre controle interno e gestão de riscos no EnANPAD e Congresso USP: análise bibliométrica no período 2001-2011. Revista Universo Contábil, v. 9, n. 4, p. 29-49, 2013.

SHAVELL, Steven. Suit, settlement, and trial: A theoretical analysis under alternative methods for the allocation of legal costs. The Journal of Legal Studies, v. 11, n. 1, p. 55-81, 1982.

SOIN, Kim; COLLIER, Paul. Risk and risk management in management accounting and control. University of Exeter, Open Research Exeter (ORE), 2013.

TRAPP, Adriana Cristina Garcia; CORRAR, Luiz. Avaliação e gerenciamento do risco operacional no Brasil: análise de caso de uma instituição financeira de grande porte. Revista Contabilidade \& Finanças, v. 16, n. 37, p. 24-36, 2005. 